\title{
Success of frozen embryo transfer: Does the type of gonadotropin influence the outcome?
}

This article was published in the following Dove Press journal:

International Journal of Women's Health

II May 2010

Number of times this article has been viewed

\author{
Hesham Al-Inany' \\ Pieter van Gelder ${ }^{2}$ \\ 'Egyptian IVF-ET Center, Maadi, Egypt; \\ 2PSCT BV, Den Haag, The Netherlands
}

Correspondence: P van Gelder PSCT BV, Nootdorpse Landingslaan 292, 2496TB Den Haag, The Netherlands

Tel +3I 622955219

Fax +3 I I 536I 8930

Email p.h.a.j.m.vangelder@tudelft.nl
Objectives: To determine whether there is a difference in outcome between different ovulationinduced cycles after frozen-thawed embryo transfer (FET).

Methods: We searched the Cochrane Menstrual Disorders and Subfertility Group's trials register in May 2009, the Cochrane Central Register of Controlled Trials (Cochrane Library, Issue 1, 2008), ISI Web of Knowledge (1985 to August 2009), and reference lists of articles. Relevant conference proceedings were hand-searched and researchers in the field were contacted. Randomized controlled trials and retrospective studies were included, comparing the various cycle regimens and different methods during FET in assisted reproductive technology, ie, in vitro fertilization and intracytoplasmic sperm injection.

Results: Using the agonist long protocol for downregulation, five trials provided extractable data for live-birth rates, ongoing pregnancy, and clinical pregnancy rates following FET. One trial provided extractable data for clinical pregnancy rate. There was no evidence of a significant difference in any outcome between the users of urinary gonadotropins versus recombinant follicle-stimulating hormone. Data on implantation and miscarriage rates following FET were not available for analysis.

Conclusions: It seems that clinical pregnancy rate after FET is not influenced by the type of gonadotropins used. Research should be directed towards improving freezing and thawing techniques.

Keywords: infertility, assisted reproductive technology, frozen embryo transfer, gonadotropins

\section{Introduction}

Frozen-thawed embryo transfer (FET) has become a vital aspect of assisted reproduction. ${ }^{1}$ Pregnancy rates following FET treatment have always been lower than following fresh embryo transfer. Nevertheless, FET increases the cumulative pregnancy rate, reduces cost, is relatively simple to undertake, and can be accomplished in a shorter time period compared with repeated "fresh" cycles. FET is performed using different cycle regimens, ${ }^{2}$ ie, spontaneous ovulatory cycles, cycles in which ovulation is induced by drugs, and cycles in which the endometrium is artificially prepared using oestrogen and progesterone hormones, with or without a gonadotropin-releasing hormone $(\mathrm{GnRH})$ agonist. Results of fresh and FET in women undergoing in vitro fertilization (IVF) with GnRH agonists and GnRH antagonists showed no significant difference between methods. ${ }^{3}$

Little is known about the impact of type of gonadotropins on FET. In the medical literature, recent meta-analyses have shown a significant advantage of using urinary gonadotropins over recombinant follicle-stimulating hormone (rec-FSH) during ovarian 
stimulation, with higher clinical pregnancy and live-birth rates in the former group. ${ }^{4,5}$ However, these reviews did not directly assess the results of using different FSH preparations in FET cycles, nor cumulative pregnancy and live-birth rates per stimulated cycle.

The beneficial effect of urinary gonadotropins has been investigated in the Menotropin versus Recombinant FSH in vitro Fertilisation Trial (MERiT), which showed differences in embryo quality and endometrial receptivity between rec-FSH and highly purified human chorionic gonadotropin (HP-hMG). ${ }^{6}$ Balasch et al ${ }^{7}$ compared follicular development and estradiol levels after ovarian stimulation in pituitary-suppressed, normally ovulating women undergoing IVF, using highly purified urinary FSH (u-FSH-HP) and rec-FSH. A secondary variable in their study was embryo implantation potential, which is closely related to appropriate follicular development and oocyte competence. They found that rec-FSH is more effective than $\mathrm{u}-\mathrm{FSH}-\mathrm{HP}$ when used in the same patient for inducing multiple follicular development in downregulated cycles, as indicated by ovarian performance and oocyte maturity. In addition, they concluded that rec-FSH yields significantly higher implantation rates than u-FSH-HP when used in patients undergoing their second IVF attempt.

Clinical efficiency in IVF procedures have been debated for years. Definition of a goal, or endpoint, for IVF treatments has varied between clinicians, regulatory bodies, and organizations, with some regarding a clinical pregnancy, an ongoing pregnancy, a live birth, or even a "take-home" baby as the primary outcome of IVF treatments. ${ }^{8-21}$ This controversy has recently been complicated by the use of single embryo transfer to reduce the incidence of multiple births. This has added another variable to the equation, ie, whether a multiple gestation pregnancy should be considered a success or a failure. Regardless of the exact definition of success, the use of FET has undoubtedly led to more success in assisted reproduction. The objective of this systematic review and meta-analysis was to determine the effectiveness of the use of urinary gonadotropins versus rec-FSH in FET.

\section{Materials and methods}

In order to determine the effectiveness of cryo-embryo transfers following ovarian stimulation with urinary gonadotropins compared with rec-FSH, we included comparative trials (ie, randomized controlled trials, nonrandomized controlled trials, cohort studies [prospective or retrospective], and casecontrol studies). Study designs were classified according to their methodological rigor, with lower levels of evidence included only if there was a lack of adequate higher quality evidence. Adequacy of evidence was determined after taking into consideration the number and sample sizes of the included studies at each level of evidence.

\section{Participants}

Participants in the included studies were women who were part of a couple, underwent assisted reproduction, ie, IVF or intracytoplasmic sperm injection (ICSI) for any cause of infertility, and underwent cryo-embryo transfer. Women undergoing transfer from donor oocytes and/or embryos were excluded. Duration, type (ie, primary or secondary), and cause of infertility were not considered as separate confounders, although they are presented descriptively in the overview of studies, along with all other possible confounding factors.

\section{Interventions}

Trials comparing women undergoing cryo-embryo transfer following ovarian stimulation with urinary gonadotropins versus rec-FSH were included. All dosages and durations of stimulation were included, as were different freezing and thawing techniques. Details of ovarian stimulation protocols, and freezing/thawing cycles are presented in the overview of studies.

\section{Outcome measures}

The primary outcome measures for this review were clinical pregnancy and miscarriage rates following cryo-embryonic transfer per woman. Secondary outcomes were implantation, ongoing pregnancy, and live-birth rates following cryo-embryonic transfer. Additionally, the cumulative clinical pregnancy and live-birth rates per woman were evaluated. The cumulative rates were calculated by combining the pregnancy rates from the fresh and frozen-thawed cycles, when separately presented. In a subgroup analysis, the results for each group (eg, women with fresh cycles and women with frozen-thawed cycles) were also analysed separately from each other.

\section{Search methods for identification of studies}

We electronically searched the Medline, Embase, and Central ISI Web of Knowledge databases from 1985 to August 2009, as well as the Cochrane Library, Issue 3, 2009, with no language barriers. We further performed hand searches of conference abstracts of major proceedings (eg, European Society of Human Reproduction and Embryology and American Society of Reproductive Medicine) for the past five years, and the reference lists of expert-identified major 
review articles relevant to the present review and, in turn, the reference lists of included trials. We also searched prospective trial registers of, eg, the World Health Organization (WHO), the ClinicalTrials.gov website, and the International Standard Randomized Controlled Trial Number (ISRCT) Registry, for citations of ongoing and unpublished trials.

Individual search strategies were developed for each database using the following MeSH terms, keywords, and a modified version of the Cochrane Highly Sensitive Search Strategy (HSSS): urinary, recombinant, follicle stimulating hormone, FSH, gonadotropin, gonadotropin, human menopausal gonadotropin, human menopausal gonadotropin, urinary FSH, u-FSH, hMG, recombinant FSH, rec-FSH, frozen embryo transfer, frozen embryo replacement, frozen embryo cycle, FET, clinical pregnancy rate, and brand names of drugs used in treatment.

\section{Data collection and analysis}

In this meta-analysis, live birth rate after FET was the primary parameter. Several studies only present cumulative pregnancy rates (ie, their results also included pregnancy rates for fresh embryo transfers). For sound statistical comparison, fresh and FETs should be analysed separately. The effects of urinary gonadotropins and rec-FSH on the fresh embryo transfers have already been demonstrated in many studies, ie, that there is no significant difference in pregnancy rates. To isolate the pregnancy rate for FET from the cumulative pregnancy rate, the flow diagram shown in Figure 1 can be followed.

Of all the $n$ patients ( $n_{1}$ in $u-F S H$ and $n_{2}$ in rec-FSH) who underwent controlled ovarian hyperstimulation in the study, $\mathrm{m}$ patients $\left(\mathrm{m}_{1}\right.$ in $\mathrm{u}-\mathrm{FSH}$ and $\mathrm{m}_{2}$ in rec-FSH) underwent oocyte retrieval, of whom p patients had oocytes retrieved ( $\mathrm{p}_{1}$ in $\mathrm{u}-\mathrm{FSH}$ and $\mathrm{p}_{2}$ in rec-FSH). From these patients, a total of q oocytes were obtained and inseminated via regular assisted reproductive technology (ART) procedures: $\left(\mathrm{q}_{1}\right.$ in $\mathrm{u}-\mathrm{FSH}$ and $\mathrm{q}_{2}$ in rec-FSH). The flow of oocyte/embryo assessments is illustrated in Figure 1. A total number of $r$ embryos are transferred in the fresh cycles $\left(r_{1}\right.$ in u-FSH and $\mathrm{r}_{2}$ in rec-FSH), and s embryos are cryopreserved ( $\mathrm{s}_{1}$ in $\mathrm{u}-\mathrm{FSH}$ and $s_{2}$ in rec-FSH). During a certain period, $t$ embryos are thawed ( $\mathrm{t}_{1}$ in $\mathrm{u}-\mathrm{FSH}$ and $\mathrm{t}_{2}$ in rec-FSH), of which $\mathrm{u}$ thawed embryos are transferred ( $\mathrm{u}_{1}$ in $\mathrm{u}-\mathrm{FSH}$ and $\mathrm{u}_{2}$ in rec-FSH) in $\mathrm{v}$ patients $\left(\mathrm{v}_{1}\right.$ in $\mathrm{u}-\mathrm{FSH}$ and $\mathrm{v}_{2}$ in rec-FSH) and $\mathrm{w}$ cryocycles $\left(\mathrm{w}_{1}\right.$ in $\mathrm{u}-\mathrm{FSH}$ and $\mathrm{w}_{2}$ in rec-FSH). Finally, the clinical pregnancy rate per frozen-thawed embryo cycle can be derived. In several studies, other units were used for the pregnancy rate, or even omitted. Some authors present only the live birth (or baby take-home) rates. Other authors present the data over a one-year period, instead of a cycle.

Data collection and analysis was conducted in accordance with guidelines presented in the Cochrane Handbook for Systematic Reviews of Interventions. ${ }^{22}$ A data set (N1, N2, M1, M2) was extracted from each study, in which N1 and N2 subjects in the rec-FSH and urinary gonadotropins groups, respectively, underwent a cycle during which frozen-thawed embryos were replaced, resulting in M1 pregnancies in the rec-FSH group and M2 in the urinary gonadotropins group.

Dichotomous outcomes (eg, clinical pregnancy rates) were expressed as an odds ratio (OR) with $95 \%$ confidence intervals (CI) for each study. Results from each study were meta-analysed with the Comprehensive Meta-Analysis software, version 2.2046 (Biostat, Englewood, NJ) using the Mantel-Haenszel method with a fixed-effect model.

A standardized data extraction form was developed and piloted for consistency and completeness. Trials were considered for inclusion and trial data were extracted by one review author (PVG), and checked for accuracy and completeness by a second reviewer (HAI) according to the inclusion criteria, with conflicts being resolved by consensus. In the event of missing data, corresponding authors of included studies were contacted for additional information.

The analysis of dichotomous data and continuous data was performed using a random-effects model, and the OR and weighted mean difference, respectively, evaluated with their $95 \%$ CI. $P$ values are presented for further confirmation of the results. A $P$ value of $<0.05$ was considered to be statistically significant.

In the graphical display of the analyses, an increased probability of a favorable outcome (ie, clinical pregnancy) for u-FSH would be displayed graphically to the right of the center line and vice versa. On the contrary, an increased probability of not having a detrimental outcome (eg, miscarriage) from u-FSH would be displayed graphically to the left of the center line.

\section{Unit-of-analysis issues}

Given that clinical infertility trials should ideally deal with women and not cycles, a woman should only be included once (ie, one cycle per woman) in the trial, otherwise there would be a unit-of-analysis error. Common statistical errors in the design and analysis of subfertility trials have already been reported, ${ }^{23}$ and the typical unit-of-analysis errors in fertility trials are well described. The authors found that $82 \%$ of the 39 trials which they investigated contained at least 

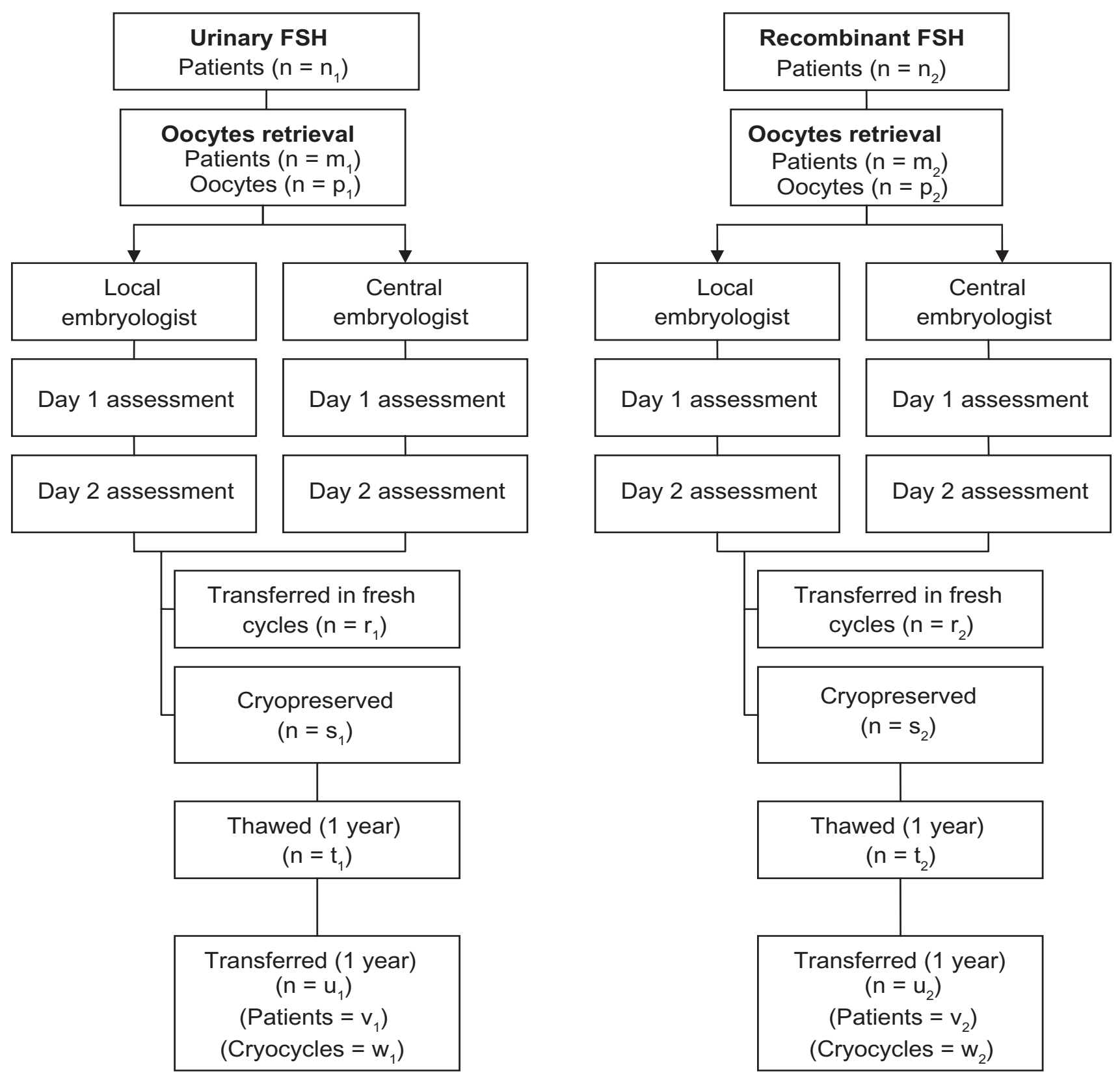

Figure I Flow diagram for data extraction from trials with frozen-thawed embryo transfers.

one unit-of-analysis error, but still passed review. Other unit-of-analysis issues have also been addressed by Van Gelder. ${ }^{24}$ Each study in the underlying meta-analysis was critically reviewed, and errors and omissions identified by Vail and Gardener ${ }^{23}$ were checked.

\section{Assessment of heterogeneity}

The classical measure of heterogeneity is Cochran's Q which is the weighted sum of squared differences between individual study effects and the pooled effect across studies. Also, the $\mathrm{H}_{\text {and }} \mathrm{I}^{2}$ statistics have been calculated and included in the assessment. A large value of Q indicates that there is significant heterogeneity between studies. The significance level for this statistic was set to 0.10 rather than the usual 0.05 . The $\mathrm{I}^{2}$ test describes the percentage of the variability in effect estimates attributable to heterogeneity rather than sampling error (chance), with an $\mathrm{I}^{2}$ value $>50 \%$ considered to represent substantial heterogeneity. ${ }^{25}$

\section{Assessment of publication bias}

Publication bias was assessed by visually noting the scatter of the point estimates and CIs for each study and by the Trim and Fill method when there were at least 10 studies in an analysis. Since the number of included studies in all of the 
analyses was below 10, the assessment was cancelled. No subgroup analysis was carried out in this meta-analysis.

\section{A priori sample size calculation}

A conventional approach was used to calculate the minimum required sample size with four parameters: Type I error, power, assumptions in the first group, and expected treatment effect. Type I error and power were fixed at the conventional levels ( $5 \%$ for Type I error, $80 \%$ for power). Assumptions related to the first group were prespecified on the basis of previously observed data (a clinical pregnancy rate of FET per randomized woman of $25 \%$ ), and an expected treatment effect of 5\% was expected to be clinically meaningful. With these assumptions, the sample size required was 1290 for group 1 and 1290 for group 2. None of the analyses reached this level of confidence because of the lack of available data in the literature.

\section{Results}

\section{Search results}

Electronic and hand searches identified 265 potentially relevant citations based on title/abstract screening. Of these, five Cochrane reviews were identified, ${ }^{2,26-29}$ two descriptive studies of FET, ${ }^{30,31}$ and one meta-analysis. ${ }^{5}$ In total, 247 citations were excluded, and 18 citations to comparative studies were further evaluated using the full-text manuscripts. ${ }^{32-49}$ Finally, only five studies met the inclusion criterion. ${ }^{37,38,42,46,48}$

Before the five trials are discussed, a brief summary is presented of a number of papers which did not fulfill the inclusion criteria, but which provide interesting data on FET.

Oehninger et $\mathrm{al}^{41}$ presented a retrospective study with more than 9000 embryos which had been cryopreserved in gonadotropin-stimulated in vitro IVF cycles. Over 1500 thaw and transfer cycles yielded a pregnancy rate of approximately $25 \%$. Different ovarian stimulation regimens (various preparations of $\mathrm{FSH}$, alone or in combination with hMG, with or without concomitant use of a GnRH agonist) did not influence embryo survival or pregnancy rate. Likewise, the application of oocyte/embryo micromanipulation techniques for assisted fertilization (ICSI for male infertility) or assisted hatching (performed selectively) did not have an impact on pregnancy results. Pregnancy outcome following embryo cryopreservation/thawing in IVF cycles stimulated with a combination of a GnRH agonist (long protocol) and FSH (urinary, urinary highly purified, or recombinant) were reported..$^{41}$
In a paper by Francsovits et al, ${ }^{34}$ a total of 389 oocytes were collected in a human follicle stimulating hormone group and 415 oocytes in an rec-FSH group. Part of the embryos were cryopreserved and clinical pregnancy rates were similar in both groups (13/35 versus 11/34, respectively).

Gerli et al, ${ }^{36}$ studied 60 IVF patients undergoing embryo transfer for the first time and randomly allocated them into two groups. Group A $(n=30)$ had subcutaneous administration of u-FSH (Fostimon 75, AMSA, Italy). Group B $(n=30)$ had subcutaneous administration of u-FSH (Metrodin 75 HP, Serono, Italy). This prospective randomized clinical study in an IVF embryo transfer program showed that both drugs were equally safe and effective. The two groups did not differ in clinical pregnancy rates per attempt and per transfer. When frozen embryo cycles were included, the difference in pregnancy rate became significant.

In a study by Salumets et $\mathrm{al},{ }^{45}$ FET was characterized by impaired pregnancy outcome and increased incidence of pregnancy loss compared with fresh IVF/ICSI embryo transfers. This study reviewed the outcome of 1242 FETs with respect to the age of the woman, the method of fertilization, embryo quality before and after freezing, and the number of embryos transferred. These data revealed that the delivery rate after FET depended on both the woman's age and the quality of embryos transferred, at the same time being unaffected by IVF/ICSI treatment. Ovarian stimulation was performed using either human menopausal or rec-FSH after suppression was achieved.

Lieberman et $\mathrm{al}^{40}$ reported that live birth rates were similar for urinary and recombinant gonadotropin preparations. They also reported that the implantation rates for frozen embryos were not significantly lower than for fresh embryos. However, no details were given about whether ovarian stimulation was performed using human menopausal or rec-FSH.

Wang et $\mathrm{al}^{47}$ presented a retrospective study analysing 3570 FET cycles (involving 1438 couples) with a view to increasing our understanding of the clinical circumstances that influence the potential for embryo implantation. The overall implantation rate was $9.1 \%$. The characteristics associated with a more favorable implantation rate were the success of the previous fresh embryo transfer cycle, age $<40$ years, and nontubal factor aetiology of infertility. However, no details were given about whether the ovarian stimulation was performed using human menopausal or rec-FSH.

Oktay et $\mathrm{al}^{50}$ carried out a meta-analysis of ICSI cycles with frozen-thawed oocytes in comparison with a matched-control group undergoing IVF-ICSI with fresh 
oocytes in a large ART program. They report that clinical and live-birth pregnancy rates per embryo transfer with oocyte cryopreservation are lower compared with success rates with fresh oocytes. However, no details were given about whether the ovarian stimulation was performed using human menopausal or rec-FSH.

Gelbaya et $\mathrm{al}^{35}$ assessed the implantation, pregnancy, and live-birth rates after FET in a natural or hormonal control cycle. Two comparable groups of women with regular menstrual cycles: group A $(n=212)$ had FET in a natural cycle after spontaneous ovulation; group B $(n=205)$ had FET in a pituitary-desensitized hormonally controlled cycle. In group B, a GnRH agonist was commenced in the mid-luteal phase of the previous cycle and discontinued three days before embryo transfer. Oral estradiol valerate and a vaginal progesterone pessary were used to prepare the endometrium. Embryo transfer was carried out three days after detection of the endogenous luteinising hormone surge in group $\mathrm{A}$ and on day three of progesterone administration in group B. In the 212 women who had natural-cycle FET, 172 embryo transfers were performed and 247 embryos replaced. The implantation rate was $14.1 \%(35 / 247)$. Twenty clinical pregnancies $(20 / 172,11.6 \%)$ were achieved. In the 205 women who had downregulated hormone replacement cycle FET, 173 embryo transfers were performed and 243 embryos replaced. The implantation rate was $13.5 \%$ (33/243). Eighteen clinical pregnancies (18/173, 10.2\%) were achieved. There were no significant differences between the two groups with regard to the implantation, clinical pregnancy, or live-birth rates per cycle or per embryo transfer. The findings of Gelbaya et $\mathrm{l}^{35}$ suggest that both FET protocols are equally effective in terms of implantation rate and pregnancy outcome in women with regular menstrual cycles. However, no details were given about whether the ovarian stimulation was performed using human menopausal or rec-FSH.

Racowksy et al ${ }^{44}$ compared the cumulative pregnancy rates for fresh and frozen-thawed embryos and found no significant difference between rec-FSH and u-FSH in clinical pregnancy rates per started cycle [44.1 (367/832) versus $43.6 \%(248 / 569)]$, or delivery rates [37.9\% (315/832) versus $35.5 \%(202 / 569)]$.

Using the agonist long protocol for downregulation, five trials provided extractable data for this review and meta-analysis. The characteristics of these trials are discussed below in detail.

Ziebe et $\mathrm{al}^{48}$ evaluated as part of a randomized, assessor-blind, multinational trial conducted in 731 women undergoing IVF after stimulation with highly purified human menopausal gonadotropin (HP-hMG; MENOPUR, $\mathrm{n}=363$ ) or rec-FSH (rec-FSH; GONAL-F, $\mathrm{n}=368$ ). Ongoing pregnancy was the primary endpoint [HP-hMG 27\% and recFSH 22\%; OR (95\% CI) 1.25 (0.89-1.75)]. The live-birth, ongoing pregnancy, and ongoing implantation rates for top quality embryos were higher with HP-hMG than rec-FSH [48 versus $32 \%(P=0.038), 48$ versus $32 \%(P=0.038)$, 41 versus $27 \%(P=0.032)$, respectively]. Both the proportion of embryos with at least $50 \%$ surviving blastomeres after cryopreservation and of embryos resuming mitosis were more frequent with HP-hMG than with rec-FSH. A one-year followup of the cryopreserved embryos included data for 178 patients (HP-hMG 89, rec-FSH 89) who had embryos thawed in the specified time period, of whom 142 patients (HP-hMG 69, rec-FSH 73) underwent 206 embryo transfer cycles (HP-hMG 93, rec-FSH 113) using 331 embryos (HP-hMG 145, rec-FSH 186). In the first cryocycle, the livebirth rate was $9 \%$ in both the HP-hMG and rec-FSH groups. The live birth rate in the cryocycles with embryo transfer was $13 \%$ for HP-hMG and $10 \%$ for rec-FSH.

Seelig et $\mathrm{al}^{46}$ carried out a retrospective study with hMG $(n=194)$ and recFSH $(n=92)$ in a long-protocol or hMG $(n=16)$ or recFSH $(n=40)$ stimulation under pituitary suppression with the GnRH antagonist cetrotide. The two-pronuclear oocytes were transferred after endometrial preparation. Implantation rates in the freeze-thaw cycles were 5.6\% (hMG) and 3.8\% (rec-FSH) with twopronuclear oocytes from the long protocol, and $7 \%$ from the antagonist cycles, irrespective of whether hMG or recFSH was used. Pregnancy rates were similar, independent of whether they resulted from the long protocol cycles with hMG (15.4\%) or recFSH (13.1\%), or from the antagonist protocol cycles with hMG $(25.0 \%)$ or recFSH $(17.5 \%)$.

Out et $\mathrm{al}^{42}$ conducted a prospective, randomized, assessor-blind, multicenter $(\mathrm{n}=18)$ study with infertile women undergoing IVF comparing rec-FSH (Org 32489, Puregon $^{\circledR}$ ) and u-FSH $\left(\right.$ Metrodin $\left.^{\circledR}\right)$. In total, 585 subjects received rec-FSH and $396 \mathrm{u}-\mathrm{FSH}$. Ongoing pregnancy rates per attempt and transfer in the rec-FSH group were $22.17 \%$ and $25.97 \%$, respectively, and in the u-FSH group, $18.22 \%$ and $22.02 \%$, respectively. These differences were not statistically significant. Ongoing pregnancy rates including pregnancies resulting from frozen-thawed embryo cycles were $25.7 \%$ for rec-FSH and $20.4 \%$ for u-FSH $(P>0.05)$. Subsequently, 117 and 73 subjects in the rec-FSH and u-FSH groups, respectively, underwent a natural cycle during which frozen-thawed embryos 
were replaced, resulting in 17 ongoing pregnancies in the rec-FSH group and five in the u-FSH group. A second "frozen embryo" cycle was done in 26 and 15 women ("new women", not coming from the previous group of 117 and 73 women, respectively, who did not become pregnant), which resulted in seven additional pregnancies (five in the rec-USH and two in the u-FSH group). Eight women had a third frozen embryo cycle and two subjects a fourth, which did not result in ongoing pregnancies. The mean number of embryos transferred in the frozen embryo cycles was 2.1 for both groups. In total, 22 additional pregnancies were obtained in the rec-FSH group, and seven in the uFSH group, resulting in cumulative ongoing pregnancy rates (adjusted for center) of 25.7\% and 20.4\% in favor of rec-FSH.

Kahn et $\mathrm{al}^{38}$ carried out a prospective, randomized, comparative, cohort study which addressed issues of pregnancy rate per couple over several cycles of treatment and the inclusion of pregnancies from frozen embryos resulting from the study cycles. In this trial, the take-home baby rate over three cycles of IVF treatment, including those babies conceived from frozen embryos, was 54/86 (62.8\%) for women using rec-FSH (follitropin-beta) and 40/60 (66.7\%) for those using u-FSH $(P=0.63)$.

Hompes et $\mathrm{al}^{37}$ carried out an open-label, prospective, randomized study in which they studied the ongoing pregnancy rates per started cycle of 629 patients, and reported rates of $26.3 \%$ and $25.2 \%$ for $\mathrm{HP}-\mathrm{hMG}$ and rec-FSH, respectively (no statistically significant difference). Within the groups, the numbers of subjects with embryo transfer in cryo-cycles 1 and 2 were 19 and 1, respectively, in the HP-hMG group and were 31 and 4, respectively, in the rec-FSH group. The numbers of ongoing pregnancies in these cryocycles were 5/20 (25\%) in the HP-hMG group and $4 / 35(11.4 \%)$ in the rec-FSH. No statistically significant differences were observed for the ongoing pregnancy rates in the cryocycles. The same applies to the cumulative ongoing pregnancy rates, cumulative delivery rate, and cumulative live-birth rate (combining results of collecting cycle and one-year outcome of cryocycles).

Figure 2 shows the results from the meta-analysis of the above five studies, which show no evidence of a significant difference between the use of $\mathrm{u}-\mathrm{FSH}$ versus rec-FSH regarding the live-birth rate (u-FSH 6/69 versus rec-FSH 7/73, OR =0.90; $95 \% \mathrm{CI}=0.29-2.82, P=0.85)$ and the ongoing pregnancy rates (u-FSH 5/73 versus rec-FSH 17/117, OR =0.43; 95\% $\mathrm{CI}=0.15-1.23, P=0.12$ ). Data on the clinical pregnancy, implantation, and miscarriage rates following FET were not available from the randomized trials. The cumulative live-birth rate did not demonstrate any significant differences between the use of u-FSH versus rec-FSH for ovarian stimulation, according to Ziebe et $\mathrm{al}^{48}$ and Hompes et $\mathrm{al}^{37}[$ (u-FSH 105/363 versus rec-FSH 91/368, OR $=1.24 ; 95 \% \mathrm{CI}=0.89-1.72$, $P=0.20)$, (u-FSH $82 / 312$ versus rec-FSH 75/317, OR = 1.15; $95 \% \mathrm{CI}=0.80-1.65, P=0.45)$, respectively].

The cumulative ongoing pregnancy rate also did not demonstrate any significant differences between the use of u-FSH versus rec-FSH for ovarian stimulation, according to Hompes et $\mathrm{al}^{37}$ and Out et $\mathrm{al}^{42}[(\mathrm{u}-\mathrm{FSH} 82 / 312$ versus rec-FSH 80/317, OR $=1.06 ; 95 \% \mathrm{CI}=0.74-1.51, P=0.76$ ), (u-FSH 150/585 versus rec-FSH 87/396, OR = 1.22; 95\% $\mathrm{CI}=0.91-1.66, P=0.19)$, respectively]. Also, the cumulative clinical pregnancy rates per woman did not demonstrate any significant differences between the use of u-FSH versus rec-FSH for ovarian stimulation, (u-FSH 20/60 versus rec-FSH 37/86, OR $=0.66 ; 95 \% \mathrm{CI}=0.33-1.31$, $P=0.24)$.

The retrospective study by Seelig et $\mathrm{al}^{46}$ provided data on the clinical pregnancy rate following the use of $\mathrm{u}-\mathrm{FSH}$ versus rec-FSH in women undergoing ovarian stimulation in agonist and antagonist cycles. For women undergoing a long agonist protocol, the clinical pregnancy rate showed no evidence of a significant difference in effect between the use of u-FSH versus rec-FSH (u-FSH 30/195 versus rec-FSH 12/92, $\mathrm{OR}=1.21 ; 95 \% \mathrm{CI}=0.59-2.49, P=0.60)$, respectively. Using the antagonist protocol, the clinical pregnancy rates were also similar between the two groups (u-FSH 4/16 versus rec-FSH 7/40, OR $=1.57 ; 95 \% \mathrm{CI}=0.39-6.34, P=0.53$, Figure 2).

\section{Discussion}

The results of this systematic review of the literature reveal no evidence of a significant difference between the clinical results following FET produced from stimulation cycles using u-FSH versus rec-FSH. It is also of importance that this review has demonstrated the dearth of information available in the literature on this subject, with most of the identified studies comparing only the transfer of embryos from the initial cycle, with minimal information regarding clinical outcomes following the transfer of frozen-thawed embryos.

Comparisons of different gonadotropin preparations regarding efficacy and safety as well as cost effectiveness are important issues in assisted reproduction. The cumulative pregnancy rate per cycle is strongly related to the number of oocytes retrieved, and therefore number of embryos available 


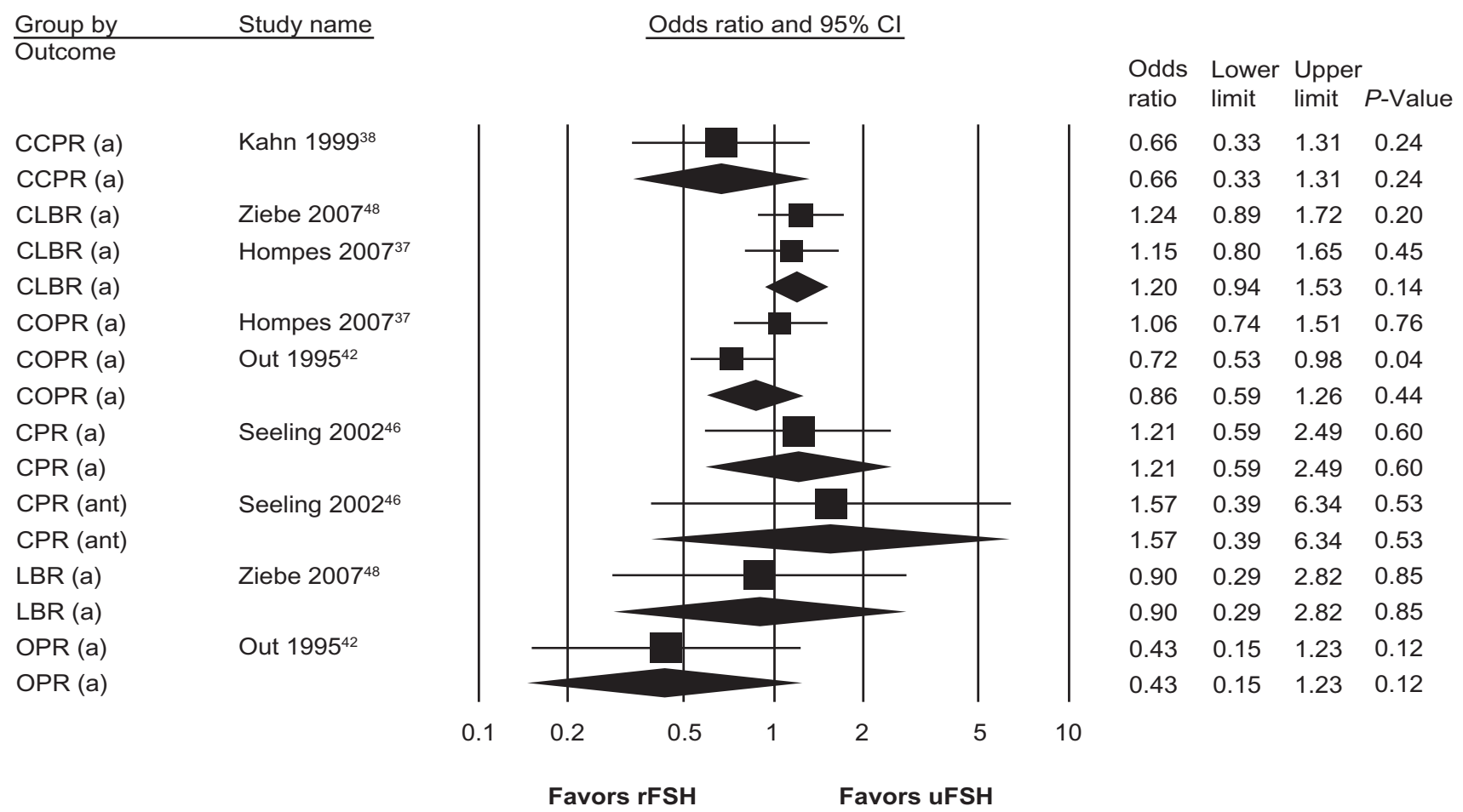

Figure 2 Forest plot demonstrating clinical outcomes.

Abbreviations: $\mathrm{Cl}$, confidence interval; LBR, live birth rate; CLBR, cumulative live birth rate; OPR, ongoing pregnancy rate; COPR, cumulative ongoing pregnancy rate; $\mathrm{CPR}$, clinical pregnancy rate; CCPR, cumulative pregnancy rate.

for transfer. ${ }^{51}$ Traditionally, ovarian stimulation protocols relied heavily on high doses of FSH to stimulate the largest cohort of follicles possible. This created two negative aspects of stimulation, a higher risk for the development of ovarian hyperstimulation syndrome, and an increased number of embryos that must either be transferred in the immediate cycle or discarded. ${ }^{52}$ Incorporation of cryopreservation of embryos has negated the latter because surplus embryos can be safely stored for future use. In centers where embryo cryopreservation is routinely used, almost $50 \%$ of all implantations are done by FET. ${ }^{53}$ Therefore, trials of FSH efficacy should include the cumulative success rates following FET per cycle started instead of only embryos transferred in the first cycle. $^{20,54}$

Over the past few years, the efficacy of different FSH preparations have been tested to determine the most effective and safe product for use in human IVF. Today, the commercially available choices can be classified into highly-purified gonadotropins, which contain both FSH and luteinizing hormone, and HP-FSH and rec-FSH preparations. Due to the advanced purification and manufacturing processes, the subcutaneous route of administration is available for all these products, which makes them more convenient and patient friendly.
In clinical trials, rec-FSH had been proposed to be clinically more efficient than u-FSH (eg, MetrodinHP, Menogon). ${ }^{55}$ However, these products have been replaced with newer compounds with a higher concentration of glycosylated FSH $\left(\right.$ Fostimon $^{\circledR}$ ) and in the case of gonadotropins, the addition of hCG. These modifications have shown that no single product has demonstrated clinical superiority over another product. ${ }^{4,5,56}$

Different aspects that may affect the implantation rate of frozen-thawed embryos have been tested over the years, including the use of GnRH agonist versus antagonist during ovarian stimulation, the type and components used during cryopreservation, and thawing techniques. ${ }^{57}$ Even so, to the best of our knowledge, and as demonstrated by the meticulous search of the literature, there are only a handful of studies that have investigated the effect of the type of FSH used during ovarian stimulation.

\section{Conclusions}

It seems that clinical pregnancy rate after FET is not influenced by the type of gonadotropins used. With respect to cumulative rates, it is noted that no significant differences in live-birth rate, ongoing pregnancy rate, and clinical pregnancy rate for fresh cycles and no significant differences 
in these rates for cryocycles implies no significant differences in the cumulative rates. Research should be directed to improving freezing and thawing techniques. At the present time there is insufficient evidence to support the use of one gonadotropin in preference to another. This review concludes that there is insufficient evidence to support the use of rec-FSH over urinary gonadotropins in ovarian stimulation prior to FET for women undergoing assisted reproductive technology.

\section{Acknowledgments}

The authors kindly acknowledge Goodlife Healthcare BV in The Netherlands for the financial support that led to this publication. The research also resulted in a systematic review, which was accepted for publication. ${ }^{58}$ There was no influence from the sponsors on the outcomes of these studies.

\section{References}

1. El-Toukhy T, Kamal A, Wharf E, et al. Reduction of the multiple pregnancy rate in a preimplantation genetic diagnosis programme after introduction of single blastocyst transfer and cryopreservation of blastocysts biopsied on Day 3. Hum Reprod. 2010;25: 418-429.

2. Ghobara T, Vandekerckhove P. Cycle regimens for frozen-thawed embryo transfer. Cochrane Database Syst Rev. 2008;CD003414.

3. Bahçeci M, Ulug U, Erden HF, et al. Frozen-thawed cleavage-stage embryo transfer cycles after previous $\mathrm{GnRH}$ agonist or antagonist stimulation. Reprod BioMed Online. 2009;18:67-72.

4. Al-Inany HG, Abou-Setta AM, Aboulghar MA, Mansour RT, Serour GI. Efficacy and safety of human menopausal gonadotropins versus recombinant FSH: A meta-analysis. Reprod Biomed Online. 2008;16:81-88.

5. Coomarasamy A, Afnan M, Cheema D, van der Veen F, Bossuyt PM, van Wely M. Urinary hMG versus recombinant FSH for controlled ovarian hyperstimulation following an agonist long down-regulation protocol in IVF or ICSI treatment: A systematic review and meta-analysis. Hum Reprod. 2008;23:310-315.

6. Afnan M. Identifying real differences in live birth rates between $\mathrm{HMG}$ and rFSH in IVF. Reprod Biomed Online. 2009;18 Suppl 2:25-30.

7. Balasch J, Fábregues F, Creus M, et al. Follicular development and hormonal levels following highly purified or recombinant follicle-stimulating hormone administration in ovulatory women undergoing ovarian stimulation after pituitary suppression for in vitro fertilization: Implications for implantation potential. $J$ Assist Reprod Genet. 2000;17:20-27.

8. Bhattacharya S, Templeton A. What is the most relevant standard of success in assisted reproduction? Redefining success in the context of elective single embryo transfer: Evidence, intuition and financial reality. Hum Reprod. 2004;19:1939-1942.

9. Buckett W, Tan SL. What is the most relevant standard of success in assisted reproduction? The importance of informed choice. Hum Reprod. 2004;19:1043-1045.

10. Davies MJ, Wang JX, Norman RJ. What is the most relevant standard of success in assisted reproduction? Assessing the BESST index for reproduction treatment. Hum Reprod. 2004;19:1049-1051.

11. Dickey RP, Sartor BM, Pyrzak R. What is the most relevant standard of success in assisted reproduction?: No single outcome measure is satisfactory when evaluating success in assisted reproduction; both twin births and singleton births should be counted as successes. Hum Reprod. 2004;19:783-787.
12. Germond M, Urner F, Chanson A, Primi MP, Wirthner D, Senn A What is the most relevant standard of success in assisted reproduction?: The cumulated singleton/twin delivery rates per oocyte pick-up: The CUSIDERA and CUTWIDERA. Hum Reprod. 2004;19:2442-2444.

13. Griesinger G, Dafopoulos K, Schultze-Mosgau A, Felberbaum R, Diedrich $\mathrm{K}$. What is the most relevant standard of success in assisted reproduction? Is BESST (birth emphasizing a successful singleton at term) truly the best? Hum Reprod. 2004;19:1239-1241.

14. Heijnen EM, Macklon NS, Fauser BC. What is the most relevant standard of success in assisted reproduction? The next step to improving outcomes of IVF: Consider the whole treatment. Hum Reprod. 2004;19:1936-1938.

15. Land JA, Evers JL. What is the most relevant standard of success in assisted reproduction? Defining outcome in ART: A Gordian knot of safety, efficacy and quality. Hum Reprod. 2004;19:1046-1048.

16. Messinis IE, Domali E. What is the most relevant standard of success in assisted reproduction? Should BESST really be the primary endpoint for assisted reproduction? Hum Reprod. 2004;19:1933-1935.

17. Min JK, Breheny SA, MacLachlan V, Healy DL. What is the most relevant standard of success in assisted reproduction? The singleton, term gestation, live birth rate per cycle initiated: The BESST endpoint for assisted reproduction. Hum Reprod. 2004;19:3-7.

18. Pinborg A, Loft A, Ziebe S, Nyboe Andersen A. What is the most relevant standard of success in assisted reproduction? Is there a single 'parameter of excellence'? Hum Reprod. 2004;19:1052-1054.

19. Schieve LA, Reynolds MA. What is the most relevant standard of success in assisted reproduction?: Challenges in measuring and reporting success rates for assisted reproductive technology treatments: What is optimal? Hum Reprod. 2004;19:778-782.

20. Tiitinen A, Hyden-Granskog C, Gissler M. What is the most relevant standard of success in assisted reproduction?: The value of cryopreservation on cumulative pregnancy rates per single oocyte retrieval should not be forgotten. Hum Reprod. 2004;19:2439-2441.

21. Wennerholm UB, Bergh C. What is the most relevant standard of success in assisted reproduction? Singleton live births should also include preterm births. Hum Reprod. 2004;19:1943-1945.

22. Higgins J, Green S. Cochrane Handbook for Systematic Reviews of Interventions. Version 5.0.1. The Cochrane Collaboration. 2008.

23. Vail A, Gardener E. Common statistical errors in the design and analysis of subfertility trials. Hum Reprod. 2003;18:1000-1004.

24. Van Gelder PHAJM. Sequential methods and reassessment of significance in Out's study, editors. Vansteelandt S, et al. In: Book of Abstracts of the International Biometric Society Channel Network Conference. April 6-9, 2009, Ghent, Belgium, 2009.

25. Higgins JP, Thompson SG, Deeks JJ, Altman DG. Measuring inconsistency in meta-analyses. BMJ. 2003;327:557-560.

26. Bayram N, van Wely M, Van der Veen F. Recombinant FSH versus urinary gonadotropins or recombinant FSH for ovulation induction in subfertility associated with polycystic ovary syndrome. Cochrane Database of Systematic Reviews. Chichester: John Wiley \& Sons, Ltd.; 2001.

27. Cantineau Astrid EP, Cohlen Ben J. Ovarian stimulation protocols (anti-oestrogens, gonadotropins with and without GnRH agonists/antagonists) for intrauterine insemination (IUI) in women with subfertility. Cochrane Database of Systematic Reviews. Chichester: John Wiley \& Sons, Ltd.; 2007.

28. Daya S, Gunby JL. Recombinant versus urinary follicle stimulating hormone for ovarian stimulation in assisted reproduction cycles. Cochrane Database of Systematic Reviews. Chichester: John Wiley \& Sons Ltd.; 2006.

29. Van Wely M, van der Veen F. Meta-analysis of recombinant FSH and urinary-derived gonadotropins for IVF or ICSI. Hum Reprod. 2003;18:1554-1555; author reply 1555-1556.

30. Hayden CJ, Balen AH, Rutherford AJ. Recombinant gonadotropins. Br J Obstet Gynaecol. 1999;106:188-96.

31. Zwart-van Rijkom JE, Broekmans FJ, Leufkens HG. From HMG through purified urinary FSH preparations to recombinant FSH: A substitution study. Hum Reprod. 2002;17:857-865. 
32. Devroey P, Tournaye H, Van Steirteghem A, Hendrix P, Out HJ. The use of a $100 \mathrm{IU}$ starting dose of recombinant follicle stimulating hormone (Puregon) in in-vitro fertilization. Hum Reprod. 1998;13:565-566.

33. El-Toukhy T, Taylor A, Khalaf Y, et al. Pituitary suppression in ultrasound-monitored frozen embryo replacement cycles. A randomised study. Hum Reprod. 2004;19:874-879.

34. Francsovits P, Tothne A, Murber A, et al. Use of human derived FSH versus recombinant FSH results in more embryos suitable for frozen preservation. Presented at: 15th World Congress on IVF. Geneva, Switzerland, Apr 19-22, 2009.

35. Gelbaya TA, Nardo LG, Hunter HR, et al. Cryopreserved-thawed embryo transfer in natural or down-regulated hormonally controlled cycles: A retrospective study. Fertil Steril. 2006;85:603-609.

36. Gerli S, Perino M, Abate A, Costabile L, Gholami H, Vitiello L. Ovarian stimulation using a new highly purified urinary FSH: A prospective randomized clinical study. Clin Exp Obstet Gynecol. 1999;26:93-94.

37. Hompes PG, Broekmans FJ, Hoozemans DA, Schats R. Effectiveness of highly purified human menopausal gonadotropin vs recombinant follicle-stimulating hormone in first-cycle in vitro fertilization-intracytoplasmic sperm injection patients. Fertil Steril. 2008;89:1685-1693.

38. Kahn J, Sunde A, von During V, Out H. A prospective randomized comparative cohort study of either recombinant FSH (Puregon) or urinary FSH (Metrodin) in in-vitro fertilization treatment. Middle East Fertility Society Journal. 1999;4:206-214.

39. Kaluarachchi A, Wijeratne S, Nelson C, Nishantha L, Wijemanne S, Senevirathne H. Study on the outcome of transfer of frozen-thawed embryos in artificially prepared cycles with or without prior gonadotropin releasing hormone agonist treatment. In: Proceedings of the 14th World Congress on in vitro fertilization and 3rd World Congress on in vitro maturation. September 15-19, 2007, Montreal, Canada. 2007; p. 43-46.

40. Lieberman B, Falconer D, Sinha S, et al. Ovarian stimulation using step-down: Safety, efficacy and outcome. Lugano, Switzerland: IBSA Institut Biochimique SA; 2003. Available from: http://www.ibsa.ch/ lieberman.pdf.

41. Oehninger S, Mayer J, Muasher S. Impact of different clinical variables on pregnancy outcome following embryo cryopreservation. Mol Cell Endocrinol. 2000;169:73-77.

42. Out HJ, Mannaerts BM, Driessen SG, Bennink HJ. A prospective, randomized, assessor-blind, multicentre study comparing recombinant and urinary follicle stimulating hormone (Puregon versus Metrodin) in in-vitro fertilization. Hum Reprod. 1995;10:2534-2540.

43. Prades M, Golmard JL, Vauthier D, Lefebvre G, Poirot C. Can cumulative pregnancy rates be increased by freezing and thawing single embryos? Fertil Steril. 2009;91:395-400.

44. Racowsky C, Orasanu B, Hinrichsen MJ, Ginsburg ES. Embryo quality based on ovulation induction: Defining the differences. Reprod Biomed Online. 2005;11:22-25.
45. Salumets A, Suikkari AM, Makinen S, Karro H, Roos A, Tuuri T. Frozen embryo transfers: Implications of clinical and embryological factors on the pregnancy outcome. Hum Reprod. 2006;21:2368-2374.

46. Seelig AS, Al-Hasani S, Katalinic A, et al. Comparison of cryopreservation outcome with gonadotropin-releasing hormone agonists or antagonists in the collecting cycle. Fertil Steril. 2002;77:472-475

47. Wang JX, Yap YY, Matthews CD. Frozen-thawed embryo transfer: Influence of clinical factors on implantation rate and risk of multiple conception. Hum Reprod. 2001;16:2316-2319.

48. Ziebe S, Lundin K, Janssens R, Helmgaard L, Arce JC. Influence of ovarian stimulation with HP-hMG or recombinant FSH on embryo quality parameters in patients undergoing IVF. Hum Reprod. 2007;22:2404-2413.

49. Out HJ, Driessen SG, Mannaerts BM, Coelingh Bennink HJ. Recombinant follicle-stimulating hormone (follitropin beta, Puregon) yields higher pregnancy rates in in-vitro fertilization than urinary gonadotropins. Fertil Steril. 1997;68:138-142.

50. Oktay K, Cil AP, Veeck L, et al. Comparative efficiency of IVF between frozen-thawed and fresh oocytes: A meta-analysis. Conjoint Annual Meeting of the American-Society-for-Reproductive-Medicine/ Canadian-Fertility-and-Andrology-Society. Montreal, Canada Oct 15-19, 2005. Fertil Steril. 2005;84:S37.

51. Malizia BA, Hacker MR, Penzias AS. Cumulative live-birth rates after in vitro fertilization. $N$ Engl J Med. 2009;360:236-243.

52. Vloeberghs V, Peeraer K, Pexsters A, D'Hooghe T. Ovarian hyperstimulation syndrome and complications of ART. Best Pract Res Clin Obstet Gynaecol. 2009;23:691-709.

53. Edgar DH, Gook DA. How should the clinical efficiency of oocyte cryopreservation be measured? Reprod Biomed Online. 2007; 14:430-435.

54. Jones HW Jr, Veeck LL, Muasher SJ. Cryopreservation: The problem of evaluation. Hum Reprod. 1995;10:2136-2138.

55. Daya S, Gunby J. Recombinant versus urinary follicle stimulating hormone for ovarian stimulation in assisted reproduction. Hum Reprod. 1999;14:2207-2215.

56. Moustafa M, Abdelwahed A, Abosekena I, Abdelazim S, Abou-Setta A, Al-Inany H. IVF outcomes with either highly purified FSH vs recombinant FSH in down-regulated normogonadotrophic women: A prospective comparative study in a developing country and meta-analysis. The Open Women's Health Journal. 2009;3:11-15.

57. AbdelHafez F, Desai N, Abou-Setta A, Falcone T, Goldfarb J. A systematic review and meta-analysis of prospective randomized trials comparing different techniques for embryo cryopreservation. Reprod BioMed Online. 2010; In press.

58. Al-Inany HG, Van Gelder PHAJM. Effect of urinary versus recombinant FSH on clinical outcomes after frozen-thawed embryo transfers: A systematic review. Reprod BioMed Online. 2010; In press.
International Journal of Women's Health

\section{Publish your work in this journal}

The International Journal of Women's Health is an international, peerreviewed open-access journal publishing original research, reports, reviews and commentaries on all aspects of women's healthcare including gynecology, obstetrics, and breast cancer. Subject areas include: Chronic conditions (migraine headaches, arthritis, osteoporosis);

\section{Dovepress}

Endocrine and autoimmune syndromes; Sexual and reproductive health; Psychological and psychosocial conditions. The manuscript management system is completely online and includes a very quick and fair peer-review system. Visit http://www.dovepress.com/ testimonials.php to read real quotes from published authors. 